Effect of plasma shaping on performance in the National Spherical Torus

Experiment

D. A. Gates, et al.

July 27,2006

Physics of Plasmas 
This document was prepared as an account of work sponsored by an agency of the United States Government. Neither the United States Government nor the University of California nor any of their employees, makes any warranty, express or implied, or assumes any legal liability or responsibility for the accuracy, completeness, or usefulness of any information, apparatus, product, or process disclosed, or represents that its use would not infringe privately owned rights. Reference herein to any specific commercial product, process, or service by trade name, trademark, manufacturer, or otherwise, does not necessarily constitute or imply its endorsement, recommendation, or favoring by the United States Government or the University of California. The views and opinions of authors expressed herein do not necessarily state or reflect those of the United States Government or the University of California, and shall not be used for advertising or product endorsement purposes. 


\title{
Effect of plasma shaping on performance in the National Spherical Torus Experiment $^{\text {a) }}$
}

\author{
D. A. Gates, ${ }^{1, b)}$ R. Maingi, ${ }^{4}$ J. Menard,${ }^{1}$ S. Kaye,${ }^{1}$ S. A. Sabbagh, ${ }^{2}$ G. Taylor,${ }^{1}$
}

J. R. Wilson, ${ }^{1}$ M. G. Bell, ${ }^{1}$ R. E. Bell, ${ }^{1}$ S. Bernabei, ${ }^{1}$ J. Bialek, ${ }^{2}$ T. Biewer, ${ }^{1}$ W. Blanchard, ${ }^{1}$

J. Boedo, ${ }^{3}$ C. Bush, ${ }^{4}$ M. D. Carter, ${ }^{4}$ W. Choe,${ }^{5}$ N. Crocker, ${ }^{6}$ D. S. Darrow, ${ }^{1}$ W. Davis, ${ }^{1}$

L. Delgado-Aparicio, ${ }^{7}$ S. Diem, ${ }^{1}$ J. Ferron, ${ }^{8}$ A. Field, ${ }^{9}$ J. Foley, ${ }^{1}$ E. D. Fredrickson, ${ }^{1}$

R. Harvey, ${ }^{10}$ R. E. Hatcher, ${ }^{1}$ W. Heidbrink, ${ }^{11}$ K. Hill, ${ }^{1}$ J. C. Hosea, ${ }^{1}$ T. R. Jarboe, ${ }^{12}$

D. W. Johnson, ${ }^{1}$ R. Kaita, ${ }^{1}$ C. Kessel, ${ }^{1}$ S. Kubota, ${ }^{6}$ H. W. Kugel, ${ }^{1}$ J. Lawson, ${ }^{1}$

B. P. LeBlanc, ${ }^{1}$ K. C. Lee ${ }^{13}$ F. Levinton, ${ }^{14}$ J. Manickam, ${ }^{1}$ R. Maqueda ${ }^{14}$ R. Marsala,${ }^{1}$

D. Mastrovito, ${ }^{1}$ T. K. Mau ${ }^{3}$ S. S. Medley ${ }^{1}{ }^{1}$ H. Meyer ${ }^{9}$ D. R. Mikkelsen, ${ }^{1}$ D. Mueller, ${ }^{1}$ T. Munsat, ${ }^{15}$ B. A. Nelson, ${ }^{12}$ C. Neumeyer, ${ }^{1}$ N. Nishino, ${ }^{16}$ M. Ono, ${ }^{1}$ H. Park, ${ }^{1}$ W. Park ${ }^{1}{ }^{1}$ S. Paul, ${ }^{1}$ W. Peebles, ${ }^{6}$ M. Peng ${ }^{4}{ }^{4}$ C. Phillips, ${ }^{1}$ A. Pigarov, ${ }^{3}$ R. Pinsker, ${ }^{8}$

A. Ram, ${ }^{17}$ S. Ramakrishnan, ${ }^{1}$ R. Raman, ${ }^{12}$ D. Rasmussen, ${ }^{4}$ M. Redi, ${ }^{1}$ M. Rensink, ${ }^{18}$

G. Rewoldt, ${ }^{1}$ J. Robinson, ${ }^{1}$ P. Roney, ${ }^{1}$ L. Roquemore, ${ }^{1}$ E. Ruskov, ${ }^{11}$ P. Ryan, ${ }^{4}$

H. Schneider, ${ }^{1}$ C. H. Skinner, ${ }^{1}$ D. R. Smith, ${ }^{1}$ A. Sontag, ${ }^{2}$ V. Soukhanovskii, ${ }^{18}$

T. Stevenson, ${ }^{1}$ D. Stotler, ${ }^{1}$ B. Stratton, ${ }^{1}$ D. Stutman, ${ }^{7}$ D. Swain, ${ }^{4}$ E. Synakowski, ${ }^{1}$

Y. Takase,${ }^{19}$ K. Tritz, ${ }^{7}$ A . von Halle, ${ }^{1}$ M. Wade,${ }^{4}$ R. White,,${ }^{1}$ J. Wilgen, ${ }^{4}$ M. Williams, ${ }^{1}$

W. Zhu, ${ }^{2}$ S. J. Zweben, ${ }^{1}$ R. Akers, ${ }^{9}$ P. Beiersdorfer, ${ }^{18}$ R. Betti, ${ }^{20}$ and T. Bigelow ${ }^{4}$

${ }^{1}$ Princeton Plasma Physics Laboratory, Princeton University, Princeton, New Jersey 08543

${ }^{2}$ Department of Applied Physics, Columbia University, New York, New York 10027

${ }^{3}$ University of California, San Diego, California 92093

${ }_{5}^{4}$ Oak Ridge National Laboratory, Oak Ridge, Tennessee 37831

${ }^{5}$ Korea Advanced Institute of Science and Technology, Taejon 305-701, Republic of Korea

${ }^{6}$ University of California, Los Angeles, California 94524

${ }^{7}$ Johns Hopkins University, Baltimore, Maryland 21218

${ }^{8}$ General Atomics, San Diego, California 92186

${ }^{9}$ Euratom-UKAEA Fusion Associates, Abingdon, Oxfordshire OX14 3DB, United Kingdom

${ }^{10}$ CompX, Del Mar, California 92014

${ }^{11}$ University of California, Irvine, California 92967

${ }^{12}$ University of Washington, Seattle, Washington 98195

${ }^{13}$ University of California, Davis, California 95616

${ }^{14}$ Nova Photonics, Princeton, New Jersey 08540

${ }^{15}$ University of Colorado, Boulder, Colorado 80309

${ }^{16}$ Hiroshima University, Hiroshima 739-8511, Japan

${ }^{17}$ Massachusetts Institute of Technology, Cambridge, Massachusetts 02139

${ }^{18}$ Lawrence Livermore National Laboratory, Livermore, California 94550

${ }^{19}$ Tokyo University, Tokyo 113-8654, Japan

${ }^{20}$ University of Rochester, Rochester, New York 14627

(Received 27 October 2005; accepted 30 March 2006; published online 26 May 2006)

The National Spherical Torus Experiment (NSTX) has explored the effects of shaping on plasma performance as determined by many diverse topics including the stability of global magnetohydrodynamic (MHD) modes (e.g., ideal external kinks and resistive wall modes), edge localized modes (ELMs), bootstrap current drive, divertor flux expansion, and heat transport. Improved shaping capability has been crucial to achieving $\beta_{t} \sim 40 \%$. Precise plasma shape control has been achieved on NSTX using real-time equilibrium reconstruction. NSTX has simultaneously achieved elongation $\kappa \sim 2.8$ and triangularity $\delta \sim 0.8$. Ideal MHD theory predicts increased stability at high values of shaping factor $S \equiv q_{95} I_{p} /\left(a B_{t}\right)$, which has been observed at large values of the $S \sim 37[\mathrm{MA} /(\mathrm{m} \cdot \mathrm{T})]$ on NSTX. The behavior of ELMs is observed to depend on plasma shape. A description of the ELM regimes attained as shape is varied will be presented. Increased shaping is predicted to increase the bootstrap fraction at fixed $I_{p}$. The achievement of strong shaping has enabled operation with $1 \mathrm{~s}$ pulses with $I_{p}=1 \mathrm{MA}$, and for $1.6 \mathrm{~s}$ for $I_{p}=700 \mathrm{kA}$. Analysis of the noninductive current fraction as well as empirical analysis of the achievable plasma pulse length as elongation is varied will be presented. Data are presented showing a reduction in peak divertor heat load due to increasing in flux expansion. (C) 2006 American Institute of Physics.

[DOI: $10.1063 / 1.2198174]$

\footnotetext{
a) Paper FI1 2, Bull. Am. Phys. Soc. 50, 103 (2005).

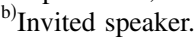




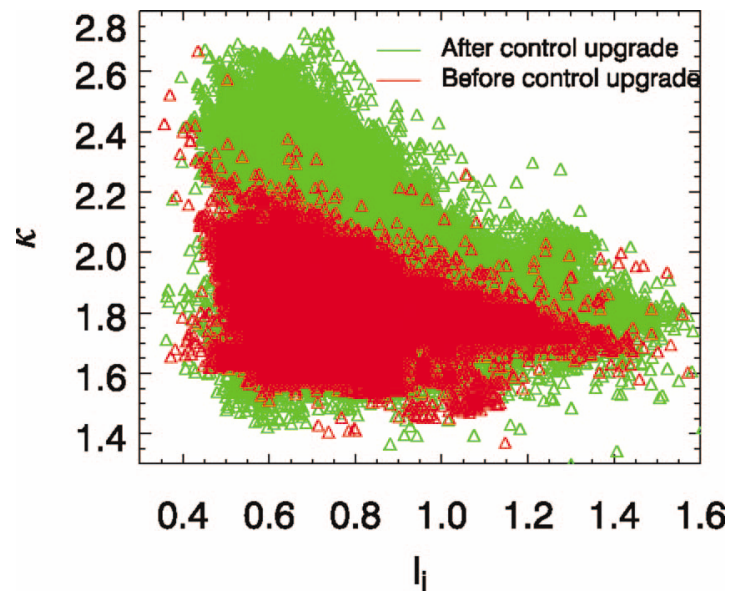

FIG. 1. (Color) Plasma elongation, $\kappa=b / a$ plotted vs normalized internal inductance, $l_{i}$ for each EFIT reconstruction in the NSTX database, filtered against rapid vertical motion. The data in green is after the control system latency reduction.

\section{INTRODUCTION}

The spherical torus $(\mathrm{ST})^{1}$ is currently being studied as a potential path to the development of fusion energy at reduced capital cost and as a potential low cost neutron source for materials testing. In addition, the study of plasmas at low aspect ratios enables the investigation of plasma physics in fusion relevant plasma regimes outside the normal operational parameter space of conventional tokamaks and thereby expands the range over which the theories that predict plasma performance can be tested. Reactor studies, such as the ARIES-ST study, ${ }^{2}$ have shown that, in order to achieve the desired high bootstrap current fraction necessary for economical operation of an ST, it is required to operate with strong plasma shaping. Similar studies for neutron sources, such as the Component Test Facility (CTF), ${ }^{3}$ also require more shaping than has been maintained in steady state in any existing device. In addition, edge localized modes (ELMs) are a serious issue for the proposed International Thermonuclear Energy Reactor (ITER) device, ${ }^{4}$ and the stability of these modes depends on plasma shape. This paper reports on efforts made on the National Spherical Torus Experiment (NSTX) to investigate the performance of plasmas that approach the shaping requirements of ST conceptual designs and to investigate the physics of plasma shaping.

\section{MACHINE IMPROVEMENTS}

In order to better investigate strongly shaped plasmas, several improvements have been made to the shape control capabilities of the NSTX. ${ }^{5}$ (These improvements were required in order to overcome the difficulties associated with maintaining shaped plasmas in steady state.) In particular, changes were made that addressed the need to control the $n=0$ vertical instability at high elongation, the need to provide $\mathrm{X}$-point control at high triangularity, and the ability to maintain constant shape as well as make fine scale shape adjustments during a discharge.

The primary method by which vertical instability control was improved was through a reduction in the latency of the

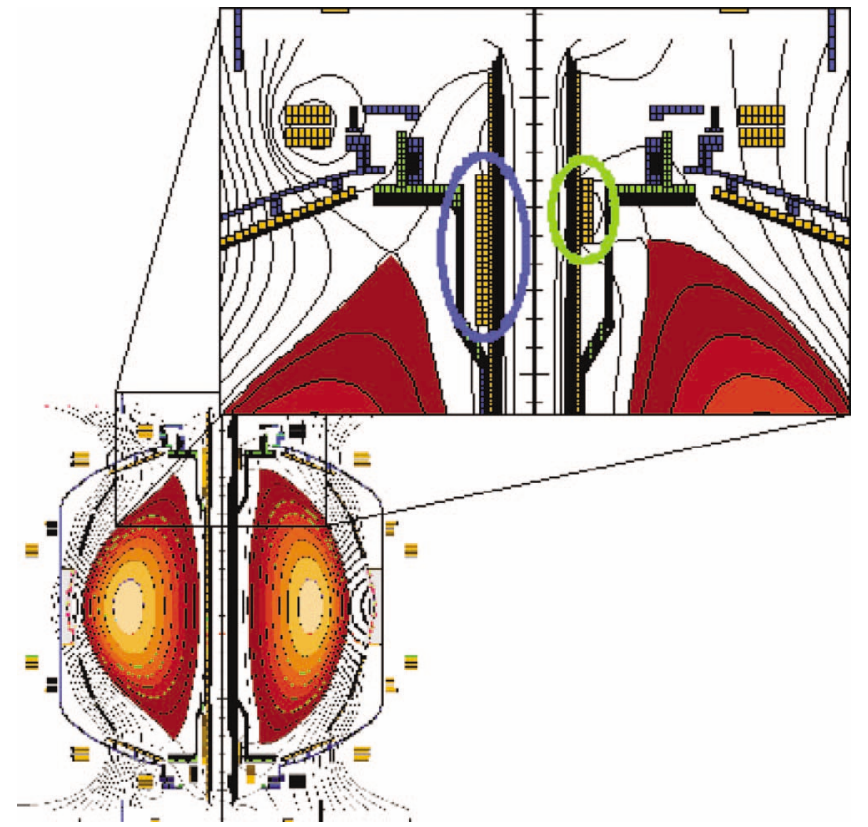

FIG. 2. (Color) The modification to the PF1A coil on NSTX has allowed simultaneous achievement of high $\kappa$ and $\delta$. The modified coil is shown on the right, and the original coil is shown on the left. Also shown are two typical high elongation double null plasmas produced using the different coils (shot 114465 on the left, 115911 on the right).

control system. The control system on NSTX is a digital computer based system that acquires 352 channels of data in real time and controls the magnet power supplies and the plasma fueling. ${ }^{6,7}$ The control system latency is the time between when a disturbance is sensed in the plasma and the time when corrective action is taken. The mean latency in the NSTX control system was measured to be $3 \mathrm{~ms}$ before the upgrades were performed. Both hardware and software optimizations were performed (each of which roughly equally improved the system latency) with a final measured latency of $0.75 \mathrm{~ms}$. The results of this improvement are summarized in Fig. 1, which shows the achieved plasma elongation ( $\kappa \equiv b / a$, where $b$ is half the vertical extent of the plasma and $a$ is half of the radial extent) as a function of the normalized internal inductance, $l_{i}$. The dependence of the instability vertical growth rate on elongation and normalized internal inductance is well documented (see, e.g., Ref. 8). Each point in the figure represents one $\mathrm{EFIT}^{9}$ reconstruction from the NSTX database. The data has been sorted to remove plasmas with excessive vertical motion on a time scale typical of vertical instability on NSTX. The red points in the figure are from discharges made before the latency reduction, while the green points are from after the reduction. For fixed $l_{i}$, the maximum achievable elongation increased by $20 \%-$ $30 \%$.

In order to achieve increased plasma triangularity, $\delta\left[\equiv\left(R_{\text {top }}-R_{\text {cent }}\right) / a\right]$ where $R_{\text {top }}$ is the major radius of the uppermost point on the plasma boundary, $R_{\text {cent }}$ is the geometric center of the plasma, and $a$ is the plasma geometric minor radius) simultaneous with high $\kappa$, it was necessary to modify the poloidal field coil arrangement on NSTX. Figure 2 shows the changes made to the PF1A poloidal field coils, which are 


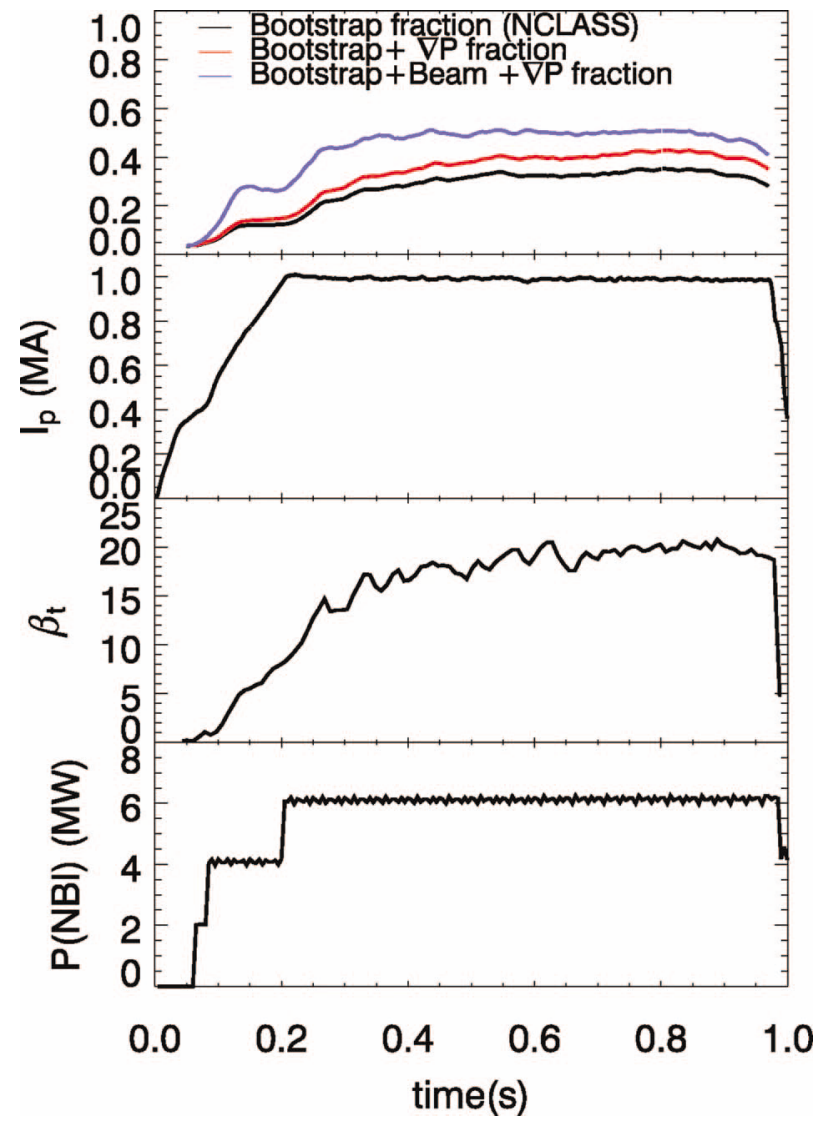

FIG. 3. (Color) Plot showing the time dependence of current sources in shot 117707. The first frame shows TRANSP calculated values of the noninductive current drive terms: the bootstrap current, the other pressure gradient driven currents (the diamagnetic and Pfirsch-Schluter currents), and the neutral beam driven current. The next three frames show the total plasma current, $\beta_{t}$ as calculated by EFIT, and the total injected neutral beam power.

located at the ends of the central solenoid. The height of the coil was reduced to $\sim 1 / 2$ the original height. This allows formation of an X point on the inboard side of the plasma near the top of the vacuum vessel. Also shown in Fig. 2 are examples of double-null divertor equilibria, one from before and one from after the PF1A coil upgrade. The change in the achievable triangularity is apparent.

In addition to these changes, real-time EFIT (rtEFIT) with isoflux control ${ }^{10}$ of the plasma boundary has been implemented on NSTX. rtEFIT was first used on NSTX in $2003^{11}$ and has been used to control plasmas heated by radio frequency waves for which accurate control of the gap between the plasma and the rf antenna is needed. Improvements to the accuracy and speed of the real-time equilibrium reconstructions have extended the utility of rtEFIT/isoflux control to the highest performance plasmas on NSTX. In fact, the longest 1 MA discharge ever run on NSTX was made using the rtEFIT/isoflux control algorithm. Data from such a discharge is shown in Fig. 3, which maintains $\beta_{t} \sim 20 \%$ for $\tau_{\text {flat }} \sim 2 \tau_{R}$. The implementation of rtEFIT has also enabled several detailed shape variation experiments that have offered insight in to the behavior of ELMs. These experiments will be described briefly in Sec. V.

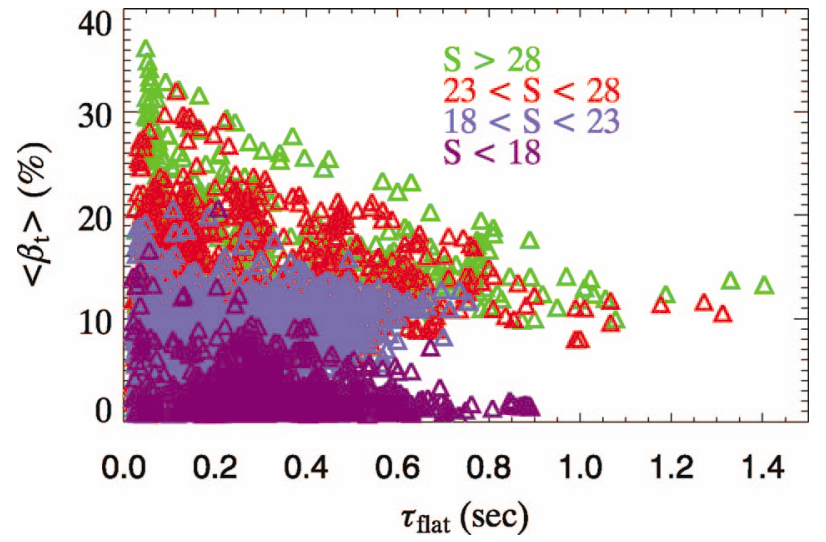

FIG. 4. (Color) Each point in the above plot represents one discharge from the NSTX database. $\left\langle\beta_{t}\right\rangle$ is $\beta_{t}$ averaged over the plasma current flattop and $\tau_{\text {flat }}$ is the flattop time over which the average is performed. The data is binned by the plasma shaping factor $S \equiv q_{95} I_{p} /\left(a B_{t}\right)[\mathrm{MA} /(\mathrm{m} \cdot \mathrm{T})]$ and bins are marked with different colors as indicated in the plot legend. There is a strong correlation between increased shape factor and increased $\beta_{t}$ and $\tau_{\text {flat }}$.

\section{GLOBAL PERFORMANCE IMPROVEMENTS}

Two of the major benefits that are associated with increased plasma shaping are an increase in the achievable $\beta_{t}$ and increased bootstrap fraction, $f_{\mathrm{bs}}$. The effect of plasma shaping on $\beta_{t}$ is presented in Fig. 4, which shows the flattop averaged $\beta_{t}$ plotted against the plasma current flattop time for the same shot. Each point in this plot represents one NSTX plasma discharge. The data are also sorted by plasma shaping factor $S\left[\equiv q_{95} I_{p} /\left(a B_{t}\right)[\mathrm{MA} /(\mathrm{m} \cdot \mathrm{T})]\right]$, where $q_{95}$ is the safety factor on the $95 \%$ flux surface with color corresponding to shape factor. As is evident from the figure, increasing the shape factor has increased both pulse length at fixed $\beta_{t}$ and $\beta_{t}$ at fixed pulse length.

To understand the benefit of increased shaping more fully, we define a figure of merit,

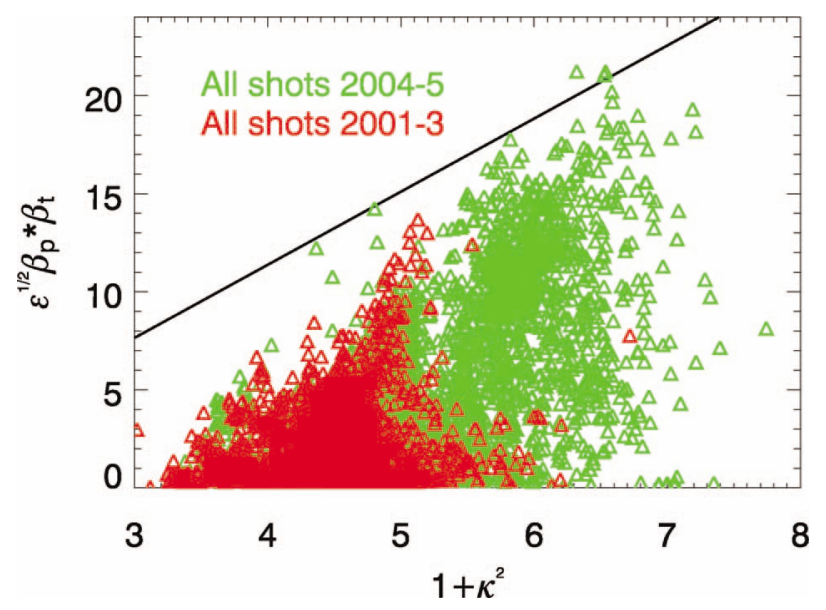

FIG. 5. (Color) Each point in the plot above represents one discharge in the NSTX database. The quantity $\sqrt{\epsilon} \beta_{p} \beta_{t}$ averaged over the plasma current flattop is plotted against $1+\kappa^{2}$, with $\kappa$ also averaged over the current flattop. The straight line in the figure is meant to guide the eye as an upper bound and to show the rough linear relationship between the maximum achievable $\beta_{p} \beta_{t}$ and $1+\kappa^{2}$. The red points are from before the control system upgrade and the green points are from after. 


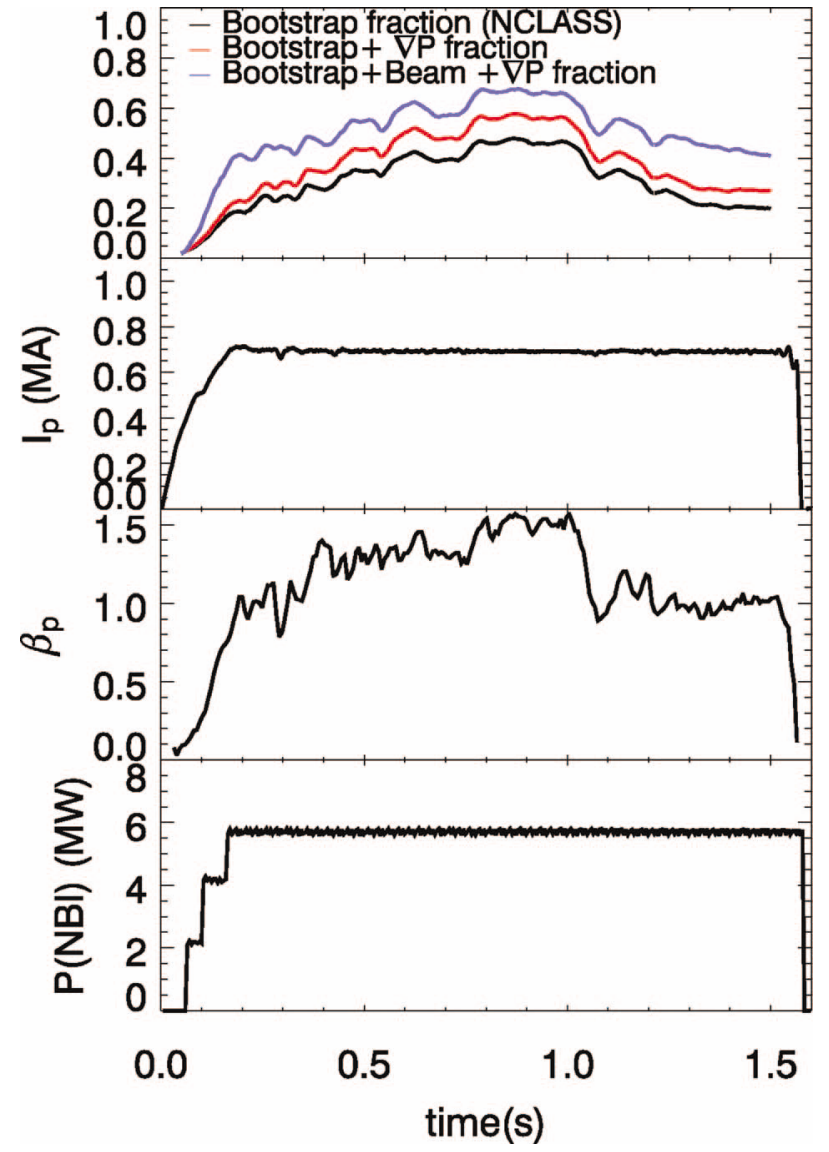

FIG. 6. (Color) Plot showing the time dependence of current sources in shot 116318. The first frame shows TRANSP calculated values of the noninductive current drive terms: the bootstrap current, the other pressure gradient driven currents (the diamagnetic and Pfirsch-Schluter currents), and the neutral beam driven current. The next three frames show the total plasma current, $\beta_{p}$ as calculated by EFIT, and the total injected neutral beam power.

$$
\beta_{\mathrm{sus}} \equiv f_{\mathrm{bs}} \beta_{t} \sim C_{\mathrm{bs}} \sqrt{\epsilon} \beta_{p} \beta_{t} \sim\left(C_{\mathrm{bs}} / \sqrt{\epsilon}\right)\left(1+\kappa^{2}\right) / 2\left(\beta_{N} / 2\right)^{2}
$$

where $C_{\mathrm{bs}} \sim 0.5$ is the coefficient that approximately relates the bootstrap fraction to $\sqrt{\epsilon} \beta_{p}$ and where use has been made of an elliptical approximation for the plasma boundary. This figure of merit, which has been previously identified in reference, ${ }^{12}$ has the benefit that it balances the inherent tradeoff between $\beta_{t}$ and bootstrap fraction (and hence pulse length) that is apparent in Fig. 4. The approximation above clearly shows that for fixed plasma elongation $\kappa$ with constant $\beta_{N}, f_{\text {bs }}$ competes directly with $\beta_{t}$. To verify experimentally the general scaling of the relationship between approximate expressions given above, we plot $\beta_{p} \beta_{t}$ vs $1+\kappa^{2}$ in Fig. 5. We have neglected the $\epsilon$ scaling in the above relationship for the plot, since the NSTX database does not contain a meaningful variation in inverse aspect ratio. As in Fig. 1, red points are for time before the control system upgrade and green is after. The data in Fig. 5 are averaged over the plasma current flattop, so each point represents one NSTX discharge. A separate analysis of the validity of the approximate expression $f_{\mathrm{bs}} \sim \sqrt{\epsilon} \beta_{p}$ has been performed using the TRANSP code with a smaller database and the relationship is valid within $10 \%-20 \%$. The deviation between the approxi-

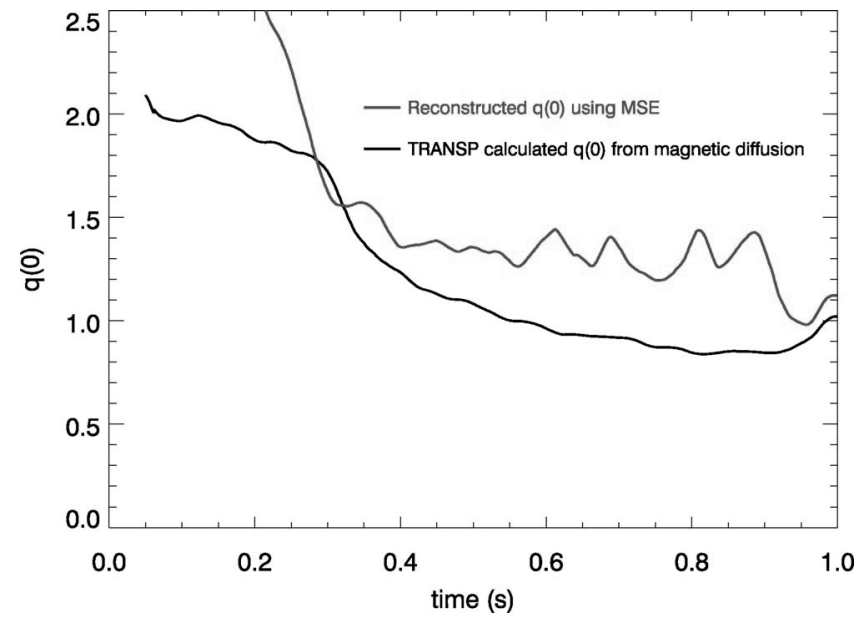

FIG. 7. Evolution of the central safety factor $q(0)$ for shot 117814 , as determined by equilibrium reconstructions made using internal magnetic pitch angle measurements from motional Stark effect polarimetry (red). Also shown is the predicted evolution of $q(0)$ calculated using the measured $T_{e}$ and $Z_{\text {eff }}$ profiles (black).

mation and the TRANSP ${ }^{13}$ calculated value of the bootstrap current can be attributed to the fast particle pressure in the plasma, which does not contribute to the bootstrap current, but which does contribute to total pressure.
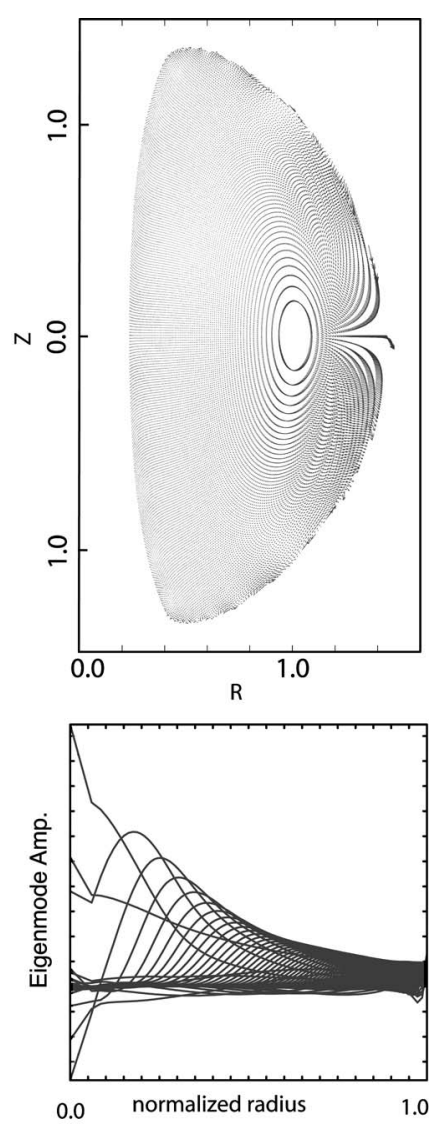

FIG. 8. Calculated unstable $n=1$ external kink eigenfunction for a typical equilibrium from shot 117707 . The plasma displacement vectors are shown in the arrows in the upper part of the figure. The lower figure shows the radial eigenfunction for individual poloidal harmonics for the same equilibrium. 


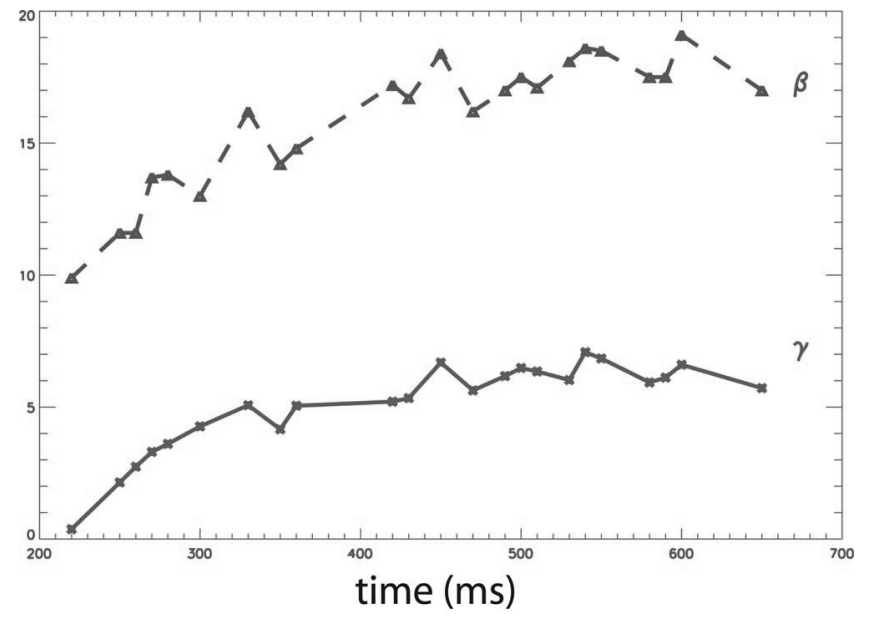

FIG. 9. Time history of the calculated growth rate for the eigenmode show in Fig. 8 for shot 117707. Also shown in the figure is the time history of $\beta_{t}$ as calculated by the EFIT code. There is clearly a correlation between the calculated growth rate and $\beta_{t}$. The plasma is calculated to be stable in the presence of a conformal perfectly conducting wall.

\section{LONG PULSE DISCHARGES}

As a result of plasma shaping, NSTX has been able to operate with longer pulse length. In particular, discharges with pulse lengths of $\sim 1.6 s$ have been achieved. This corresponds to $\sim 50 \tau_{E}$, where $\tau_{E}$ is the energy confinement time and, more importantly, to $\sim 6 \tau_{R}$, where $\tau_{R} \equiv \mu_{0} \sigma_{N C} a^{2} / 6$ is the resistive diffusion time for the first radial moment of the current profile. ${ }^{14} \sigma_{\mathrm{NC}} \sim 2 R_{0}\left(I_{p}-I_{\text {noninductive }}\right) /\left(V_{\text {loop }} a^{2} \kappa\right)$ is the neoclassical electrical conductivity. These discharges have high noninductive current fractions of $\sim 60 \%$, with $\sim 85 \%$ of the noninductive current being due to pressure driven currents. The noninductive current fraction in this case consists of the sum of the neutral beam driven current, the bootstrap current, the diamagnetic current, and the Pfirsch-Schluter current divided by the total plasma current. Figure 6 shows the time history of the noninductive current fraction as well as other plasma parameters for such a long pulse discharge. The normalized $\beta, \beta_{N}\left(\equiv \beta_{t} a B_{t} / I_{p}\right)$ is calculated to be well above the no-wall limit of $\beta_{N} \sim 4$ for the first $1 \mathrm{~s}$ of the current flattop, until the onset of a large $n=1$ mode that reduces the confinement of both the thermal plasma as well as the fast ions that heat the plasma. An interesting aspect of this particular discharge is that the pulse length is determined by the thermal limits of the toroidal field coil rather than instabilities or the available flux in the ohmic transformer. In addition to the very long pulse discharges described in the preceding paragraph, plasmas with higher current of $I_{p}=1 \mathrm{MA}$ and hence higher $\beta_{t} \sim 20 \%$ have been sustained for $2 \tau_{R}$. These discharges have elevated $q(0)$ for a time that is long compared to that expected from diffusion calculations. In particular, shot 117814, shown in Fig. 7, maintained $q(0)$ elevated above 1 for $700 \mathrm{~ms}$. The figure also shows the $q(0)$ evolution as calculated by the TRANSP code using the measured $T_{e}$ and $Z_{\text {eff. }}$ The $q(0)$ evolution is taken from equilibrium reconstructions that include $T_{e}$ and $n_{e}$ from Thomson scattering, and $J(R)$ from motional Stark effect polarimetry.

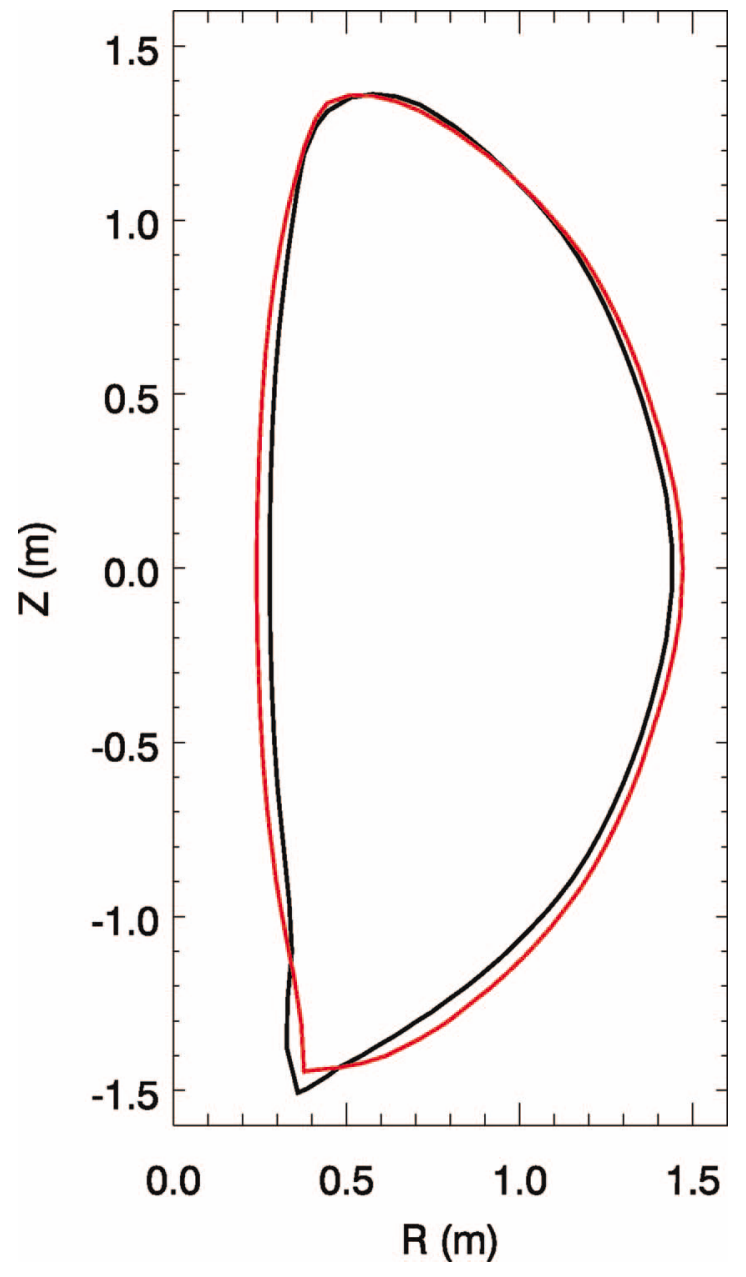

FIG. 10. (Color) Figure showing the time history of plasma parameters for shots 112581 from 2004 (black) and shot 117707 from 2005 (red). Note the dramatic change in ELM behavior.

The relationship of these discharges to the so-called "hybrid mode" of operation is under investigation.

\section{GLOBAL MHD STABILITY}

The global $n=1$ stability of a long pulse discharge has been analyzed in detail. The PEST code ${ }^{15}$ has been used to calculate the ideal MHD stability of discharge number 117707. The plasma is found to be unstable to an $n=1$ ideal pressure driven kink instability. The mode eigenfunction is shown in Fig. 8. As can be seen in the figure, the mode is global with displacement amplitude largest on the outboard midplane. The time history of the ideal growth rate is shown in Fig. 9 along with the time history of the plasma $\beta$. The plasma is stable for times before $0.2 \mathrm{~s}$ and then, as the $\beta$ rises, becomes unstable remaining so until the discharge ends. The mode is calculated to be stable in the presence an ideal conducting wall. Since the plasma is rotating rapidly, it is reasonable to invoke wall stabilization as the mechanism for violating the no-wall $\beta$ limit. The plasma equilibria used as inputs for the stability calculations were fitted to the measured pitch angle profile from motional Stark effect measurements, which have been corrected for radial electric field measurements using the toroidal velocity as measured by 


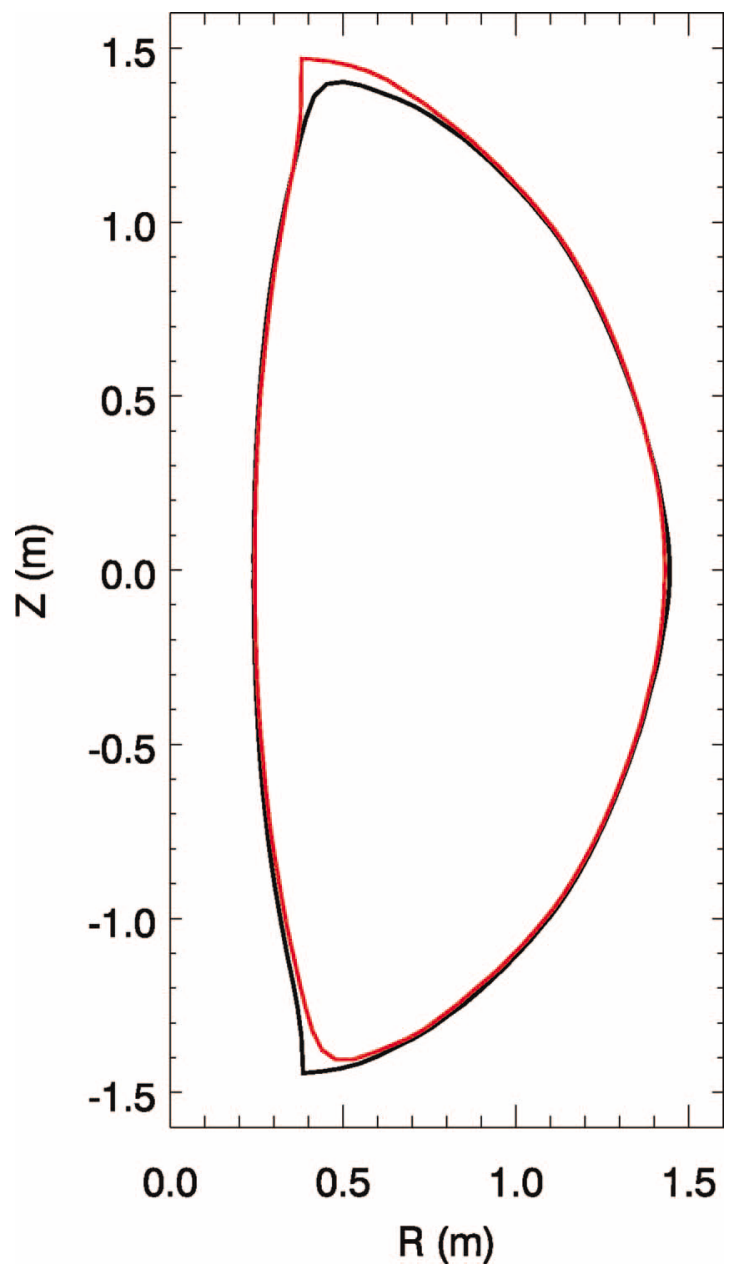

FIG. 11. (Color) Overlay of the plasma boundaries for shots 117424 (black, lower single null) and 117425 (red, upper single null).

charge exchange. In addition, the electron temperature profile, as measured by multipulse, multipoint Thomson scattering, was used as a constraint on the flux distribution.

\section{ELM CHARACTERISTICS}

An important aspect of plasma performance that is associated with changes in plasma shaping is the behavior of ELMs. The ELM characteristics of lower triangularity high elongation long pulse discharges from the 2004 run (see, e.g., Ref. 16) were not optimal, in that the ELMS from these discharges were large and had a measurable impact on the global energy confinement time. Long pulse discharges from 2005, which were run using the modified PF1A coils and had higher triangularity, had substantially improved ELM characteristics. The ELMs were smaller and, as a result, confinement was improved. Figure 10 shows a comparison between shot 112581 from 2004 and shot 117707 from 2005 demonstrating this change in ELM regime. Unfortunately, since there was not a controlled experiment to systematically vary the plasma boundary in the long-pulse regime, it is difficult to conclude definitively that the shape changes are responsible for the change in ELM behavior. Other changes to dis-

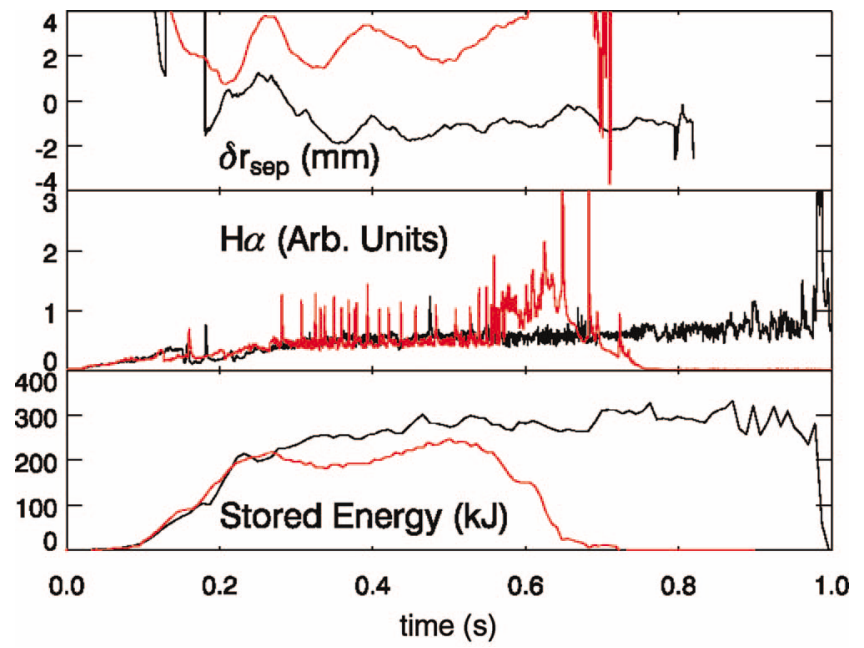

FIG. 12. (Color) The time history of plasma parameters for shots 117424 and 117425 (same shots as in Fig. 11). The dramatic change in ELM behavior substantially changes the plasma performance. Also plotted is the parameter $\delta r_{\text {sep }}$ in millimeters.

charge conditions, such as the differing beam power or different vessel wall conditions, may also be responsible for the observed changes.

A systematic experiment was performed to examine the effect on ELM behavior of changing the divertor configuration from lower single null through balanced double null to an upper single null. Similar experiments have been performed in the past to look at the H-mode power threshold. ${ }^{17}$ This change is characterized by the $\delta r_{\text {sep }}$ shape parameter defined as the distance between the flux surfaces that pass through the $\mathrm{X}$ points as measured at the outboard midplane. This study was carried out using the rtEFIT/isoflux shape control algorithm. This experiment demonstrated that small changes to the plasma boundary can indeed influence the ELM behavior in a dramatic way. $\delta r_{\text {sep }}$ was systematically

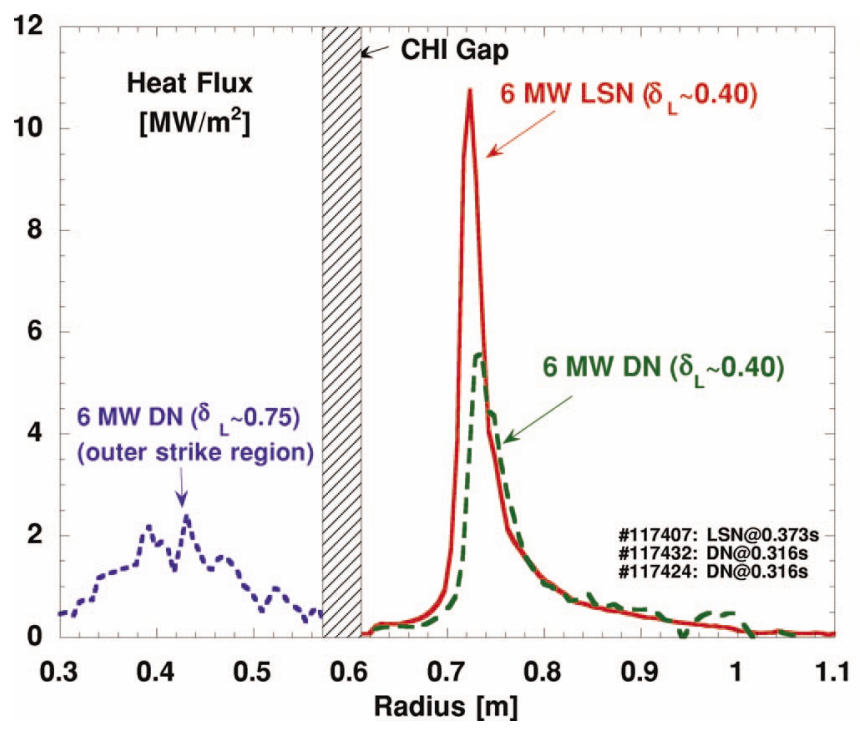

FIG. 13. (Color) The peak heat flux vs major radius for three different divertor configurations: (1) low $\delta$ lower single null, (2) low $\delta$ double null, and (3) high $\delta$ double null. All three configurations had identical heating power. The peak heat flux reduces by a factor of $\sim 5$. 
varied between +2 and $-2 \mathrm{~mm}$ (positive $\delta r_{\text {sep }}$ corresponds to an upper single null and negative to lower single null). Lower single-null plasmas had substantially smaller ELMs and exhibited energy confinement times $\sim 1.5$ times higher than upper single-null plasmas. The characteristics of these small ELMs are described in Ref. 18. This change in ELM behavior was reproducible over several shots. An example of consecutive shots with otherwise fixed parameters is shown in Fig. 11. The plasma boundaries are overlaid for comparison. Also shown in the Fig. 12 are the time histories of $\delta r_{\text {sep }}$ as determined from equilibrium reconstructions. Note the scale of variation is in millimeters. This experiment demonstrates the power of precise boundary control in creating new research opportunities, which in turn can offer greater insight into the physics of ELMs. ELMs have been shown to be a crucial issue for ITER and other future burning plasma experiments.

\section{DIVERTOR POWER LOADING}

Controlling peak heat flux is a critical issue for ITER and other burning plasma experiments, including potential future ST experiments. An additional benefit of high triangularity at low aspect ratio is strong divertor flux expansion. In lower flux expansion regimes, the divertor power loading on NSTX is similar to that expected for ITER as first shown in Reference 19. Figure 13 shows the effect of varying plasma shape on divertor power loading. Each shot in the figure has identical heating power, but by changing from low triangularity $\delta \sim 0.4$ single null to low triangularity double null to high triangularity $\delta \sim 0.8$ double null, the peak heat flux is successively reduced from $\sim 10$ to $\sim 5$ to $\sim 2.5 \mathrm{MW} / \mathrm{m}^{2}$. The ability to study heat flows in varying divertor geometries is a powerful tool for understanding the impact of flux expansion on peak heat flux.

\section{SUMMARY}

As expected, plasma shape has had a substantial impact on plasma performance on NSTX. The topics discussed in the current paper, namely $\beta$ limits, plasma pulse length, bootstrap current fraction, ELM behavior, and divertor power loading, are just a subset of the important physics issues that are affected by the shape of the plasma boundary. Boundary shape control has the distinct benefit of being an existing field of study with precise tools and detailed theories available to compare to experiment. Understanding the effect of plasma shaping in regimes that have not yet been explored represents a prime opportunity to validate and expand the utility of these theories in ways that are not possible in conventional operational regimes.

\section{ACKNOWLEDGMENT}

This work was supported by the U.S. Department of Energy Grant under contract No. DE-AC02-76CH03073.

${ }^{1}$ Y.-K. M. Peng and D. J. Strickler, Nucl. Fusion 26, 769 (1986).

${ }^{2}$ F. Najmabadi and the ARIES Team, Fusion Eng. Des. 65, 143 (2003).

${ }^{3}$ Y.-K. M. Peng, P. J. Fogarty, T. W. Burgess et al., Plasma Phys. Controlled Fusion 47, B263 (2006).

${ }^{4}$ ITER Physics Basis, Chapter 3, Nucl. Fusion 39, 2251 (1999).

${ }^{5}$ M. Ono, S. M. Kaye, Y.-K. M. Peng et al., Nucl. Fusion 40, 557 (2000).

${ }^{6}$ D. A. Gates, D. Mueller, C. Neumeyer, and J. R. Ferron, IEEE Trans. Nucl. Sci. 47, 222 (2000), Part 1.

${ }^{7}$ D. A. Gates, J. R. Ferron, M. Bell et al., "Status of the control system on the National Spherical Torus Experiment (NSTX)," Fus. Eng. Design (to be published).

${ }^{8}$ F. Hofmann, J. M. Moret, and D. J. Ward, Nucl. Fusion 38, 1767 (1998).

${ }^{9}$ L. L. Lao, H. St. John, R. D. Stambaugh, and W. Pfeiffer, Nucl. Fusion 25, $1421(1985)$

${ }^{10}$ J. R. Ferron, M. L. Walker, L. L. Lao, H. E. St. John, D. A. Humphreys, and J. A. Leuer, Nucl. Fusion 38, 1055 (1998).

${ }^{11}$ D. A. Gates, J. R. Ferron, M. Bell et al., Nucl. Fusion 46, 17 (2006).

${ }^{12}$ T. K. Mau, S. C. Jardin, C. E. Kessel et al., Proceedings of the 18th IEEE/NPSS Symp. on Fusion Engineering (Albuquerque, NM, 1999).

${ }^{13}$ R. J. Hawryluk, in Physics of Plasmas Close to Thermonuclear Conditions (CEC, Brussels, 1980), Vol. 1, p. 19.

${ }^{14}$ D. Mikkelson, Phys. Fluids B 1, 333 (1989).

${ }^{15}$ R. C. Grimm, J. M. Greene, and J. L. Johnson, in Methods in Computational Physics, edited by J. Killeen (Academic, New York, 1976), Vol. 16, p. 253.

${ }^{16}$ S. Kaye, M. G. Bell, R. E. Bell et al., Nucl. Fusion 45, S168 (2005).

${ }^{17}$ R. Maingi, C. E. Bush, E. D. Fredrickson et al., Nucl. Fusion 45, 245 (2005).

${ }^{18}$ R. Maingi, K. Tritz, E. D. Fredrickson et al., Nucl. Fusion 45, 264 (2005).

${ }^{19}$ R. Maingi, M. G. Bell, R. E. Bell et al., Nucl. Fusion 43, 969 (2003).

This work was performed under the auspices of the U.S. Department of Energy by the University of California, Lawrence Livermore National Laboratory under Contract No, W-7405-eng-48. 